\title{
Identifying Appropriate Delivery of and Referral to Pulmonary Rehabilitation in Uganda: A Survey Study of People Living with Chronic Respiratory Disease and Health Care Workers
}

\author{
Winceslaus Katagira (1D \\ Amy $\mathrm{V}$ Jones $\mathbb{1}^{2,3}$ \\ Mark W Orme iD ${ }^{2,3}$ \\ Zainab K Yusuf (iD ${ }^{2,3}$ \\ Pauline Ndagire \\ Jaliah Nanyonga' \\ Richard Kasiita ${ }^{4}$ \\ Josephine N Kasolo ${ }^{5}$ \\ Ruhme B Miah ${ }^{2,3}$ \\ Michael C Steiner $\left(\mathbb{D}^{2,3}\right.$ \\ Rupert Jones (D) ${ }^{6}$ \\ Andy Barton ${ }^{2}$ \\ Bruce Kirenga' \\ Sally J Singh (D) ${ }^{2,3}$ \\ 'Makerere University Lung Institute, \\ Kampala, Uganda; ${ }^{2}$ Department of \\ Respiratory Sciences, University of \\ Leicester, Leicester, UK; ${ }^{3}$ Centre for \\ Exercise and Rehabilitation Science \\ (CERS), NIHR Leicester Biomedical \\ Research Centre - Respiratory, \\ University Hospitals of Leicester NHS \\ Trust - Leicester, Leicester, UK; \\ ${ }^{4}$ Department of Physiotherapy, Mulago \\ Hospital, Kampala, Uganda; ${ }^{5}$ Physiology \\ Department, College of Health Sciences, \\ Makerere University, Kampala, Uganda; \\ ${ }^{6}$ University of Plymouth, Plymouth, UK
}

Correspondence: Winceslaus Katagira Email wincegira@gmail.com

\begin{abstract}
Introduction: Pulmonary rehabilitation (PR) is a low cost, high impact intervention that ameliorates the disability associated with chronic respiratory diseases (CRD). PR is becoming increasingly recognized in low resource settings where the burden of CRD is rapidly increasing. To aid the implementation of PR in Uganda, we conducted a study to assess the attitudes and opinions towards PR among patients with CRD in Uganda and explore barriers faced by health care workers (HCWs) in referring to PR.
\end{abstract}

Methods: A cross-sectional study comprising two survey populations: people living with CRD and HCWs regarded as potential PR referrers and PR deliverers. This exploratory study sought initial opinions and thoughts regarding PR, as well as baseline knowledge and potential barriers faced in the referral process.

Results: Overall, 30 HCWs (53\% female, 43\% doctors) and 51 adults with CRD (63\% female) participated in the survey. Among those with CRD, the majority reported breathlessness as a major problem (86\%) and breathlessness affected their ability to do paid and unpaid work (70\%). Interest in PR was high amongst adults with CRD (92\%) with preference for a hospital-based programme $(67 \%)$ as opposed to community-based (16\%) or home-based (17\%). All HCWs considered PR important in lung disease management, but $77 \%$ do not refer patients due to a lack of information about PR. HCWs' free-text responses identified the need for training in PR, patient education and streamlining the referral process as key elements to develop successful PR referral services.

Conclusion: To successfully set up a PR service for people with CRD in Uganda, there is a great need for appropriately tailored training and education of prospective referrers about CRD and PR programs. Educating patients about the benefits of PR as well as streamlining the referral process is critical in expanding PR services across Uganda to fulfill this unmet need.

Keywords: respiratory rehabilitation, chronic chest symptoms, medical workers, enthusiasm, knowledge, training

\section{Introduction}

The burden of chronic respiratory disease (CRD) is increasing globally ${ }^{1}$ and remains the leading cause of death and disability worldwide. ${ }^{2}$ The risk factors for CRD such as indoor air pollution, respiratory infections like pulmonary tuberculosis (PTB) ${ }^{3}$ are rife in sub-Saharan Africa (SSA), but the prevalence of CRD in the region is surprisingly 
low. ${ }^{1}$ Management of CRD in SSA remains a major challenge with largely unavailable and unaffordable diagnostics and treatments. ${ }^{4}$

Pulmonary rehabilitation (PR) is an evidence-based, ${ }^{5-7}$ low-cost, non-pharmacological intervention that ameliorates the disability associated with $\mathrm{CRD},{ }^{7}$ and is frequently used in high-income countries. PR consists of supervised, individually prescribed exercise training and health education delivered to patients by a multidisciplinary team of health professionals, and has several benefits including improved mental state, ${ }^{7-9}$ functional exercise capacity, breathlessness and health-related quality of life (HRQoL). ${ }^{9-11}$ Despite well-known benefits of PR, its availability and practice in the African region is very low. ${ }^{12}$ Policy statements from the American Thoracic Society (ATS)/European Respiratory Society (ERS) recommend increasing the implementation and provision of PR worldwide through increasing health care worker (HCW) and patient knowledge and awareness of PR, increasing patient access to $\mathrm{PR}, \mathrm{HCW}$ training and education, incorporating PR into the mainstream of medical practice and making it affordable for CRD patients. ${ }^{13-15}$ Uganda is one of the few African countries with a PR program; however, the service is largely unavailable across the country with no effective PR referral system. PR training for HCWs is not mandatory in Uganda, and exposure to PR for physiotherapists is limited. As a consequence, suitable patients are not referred for PR. Currently, there is increasing interest for PR in low- and middle-income countries (LMIC) ${ }^{16,17}$ and the treatment has shown some effectiveness in people with CRD, including post-TB lung disease (PTLD). ${ }^{12}$ However, referral rates of adults with $\mathrm{CRD}$ to $\mathrm{PR}$ programs remain low among HCWs in African settings, ${ }^{18}$ and PR uptake is low and largely underprovided. ${ }^{10}$ Information on factors affecting availability or uptake of PR services in resourcepoor countries is lacking. With an increasing burden of CRDs, there is a need to implement PR that is appealing to patients, effective, deliverable and sustainable in resource poor countries. $^{10}$

To develop and sustain a PR programme in existing health service delivery structures in Uganda and SSA at large, an exploration of PR service needs and requirements is crucial. Accordingly, this survey study aimed at assessing opinions and attitudes towards PR by people with CRD and exploring barriers HCWs face in the referral process, to inform setting up PR services in Uganda and regionally across Africa.
For adults with CRD (including chronic obstructive pulmonary disease (COPD) and PTLD) in Uganda, specific aims were to assess (i) the interest in participating in PR and (ii) the preferred mode of PR. For Ugandan HCWs in regular contact with adults with $\mathrm{CRD}$, specific aims were (i) to assess the PR knowledge and (ii) to explore barriers faced in referring patients to PR.

\section{Methods}

\section{Study Design}

This was a cross-sectional survey study for HCWs who are potential PR referrers, and for adults with CRD, including COPD and PTLD in Uganda.

\section{Study Setting and Participants}

The study was conducted at the Makerere University Lung Institute (MLI) clinic, a specialist outpatient lung health clinic in Kampala, Uganda. Between 24th January 2020 and 10th August 2020, patients with CRD referred from various health facilities across the country for routine care at the MLI clinic were invited to participate in the survey. Adults (aged $\geq 18$ years) with PTLD or spirometry confirmed COPD were included in the survey. All who did not return questionnaires were excluded. Spirometry testing was performed according to the ATS/ERS guidelines ${ }^{19}$ using a Pneumotrac ${ }^{\circledR}$ spirometer with Spirotrac $^{\circledR}$ V software (Vitalograph Ltd., Buckingham, United Kingdom). Patients with PTLD had successfully completed treatment for microbiologically confirmed PTB but continued to experience chronic respiratory symptoms with radiological evidence of lung parenchymal damage.

During the same enrollment period, HCWs from different disciplines were invited to participate in the survey. All HCWs who reported being in regular contact with adults with CRD (regarded as potential PR referrers) and those who would potentially deliver PR (eg, doctors and physiotherapists) were included in the survey. Some of the HCWs were either PR providers or worked in health facilities that provide PR.

\section{Study Procedure and Data Collection People with CRD}

Survey questionnaires (Appendix 1) were distributed to a sample of people with CRD, presenting to the MLI clinic for clinical evaluation as part of routine care. Study participants were consecutively approached to participate in the survey and all who responded were included. The surveys 
took approximately 5-10 minutes to complete and were anonymized. No patient identifiable information or clinical data were collected. Paper surveys were locked away in a secure cabinet before and after data entry. Participants could either complete the survey whilst attending their clinic appointment or the completed questionnaires would be returned a few days later in person or in a sealed envelope.

The survey questionnaires asked participants about their breathlessness in regard to their work, activity levels, visiting their friends or family, and if they would be interested in participating in a treatment that would help them become more physically active and experience less breathlessness. Furthermore, they were asked to express their opinions as to how and where they would prefer to have this treatment, and how long they would be willing to spend participating in PR.

\section{HCWs}

Survey questionnaires (Appendix 2) were distributed to HCWs working in hospitals, private health facilities and in the community around central Uganda where PR services are offered. A range of HCWs were targeted, including specialist physicians, general practitioners, nurses, and physiotherapists. No personal data were collected, except for their professional background and years of experience in managing people with CRD. Data were collected from HCWs in regards to their knowledge about PR, patient-based factors that influence their decision to recommend PR, and their experience with the PR referral process. HCWs were also asked if the Medical Research Council (MRC) dyspnea scale influenced their decision to refer patients for PR. The MRC dyspnea scale is a short and easy to use self-administered questionnaire based on the sensation of breathing difficulty experienced by the patient during daily life activities. It has grades ranging from 1 (none) to 5 (almost complete incapacity), with high grades indicating high perceived respiratory disability. ${ }^{20}$

After checking for completeness of the surveys, data were entered into Microsoft Excel Worksheet, then exported to Stata 15 statistical software (StataCorp. 2017. Stata Statistical Software: Release 15. College Station, TX: StataCorp LLC) for analysis.

\section{Data Collection During the Corona Virus Disease 2019 (COVID-19) Pandemic}

Due to the ongoing worldwide pandemic of COVID-19, lockdown measures were introduced by the government of Uganda on 18th March 2020 to curb the spread of the severe acute respiratory syndrome coronavirus 2 (SARSCoV-2). The lockdowns markedly restricted movement of patients to health facilities. Although $80 \%$ of the survey data had been collected before the COVID-19 related lockdown in our country, fewer survey questionnaires were returned after the lockdown was enforced. When access to health facilities became easier as the lockdown was eased, CRD patients resumed hospital visits, and we were able to complete data collection.

\section{Sample Size and Statistical Methods}

Formal sample size calculation was not required, as this was an exploratory study designed to obtain baseline knowledge, initial opinions and thoughts regarding PR, as well as potential barriers faced in the referral process.

\section{Quantitative Methods}

The characteristics of the study population were summarized using descriptive statistics of frequency (\%), mean and standard deviation (SD), or median and inter-quartile ranges (IQR) as appropriate.

\section{Qualitative Methods}

The qualitative data collected using the open-ended questionnaire were analyzed using autonomous qualitative counting. ${ }^{21}$ Particular words or phrases were grouped together into different categories, and then the number of times they were used in relation to a specific question were counted.

\section{Ethics and Dissemination}

The study received ethical approvals from the Mulago Hospital Research and Ethics Committee (MHREC1478), Kampala, Uganda as well as the Uganda National Council for Science and Technology (SS5105). Additional ethical approval was obtained from the University of Leicester research ethics committee (United Kingdom) (Ref No. 22349). The study was conducted in accordance with the International Council for Harmonization: Good Clinical Practice guidelines and the declaration of Helsinki. All participants provided written informed consent before taking part in the study.

\section{Results}

\section{People with CRD}

A total of 75 individuals with CRD (COPD and PTLD) were approached to participate in the survey and 51 were recruited. Of the 24 participants who did not participate, 7 presented with symptoms of an acute respiratory tract infection without underlying CRD, and 17 did not return questionnaires. A total of 51 patients with CRD were recruited (Figure 1). 


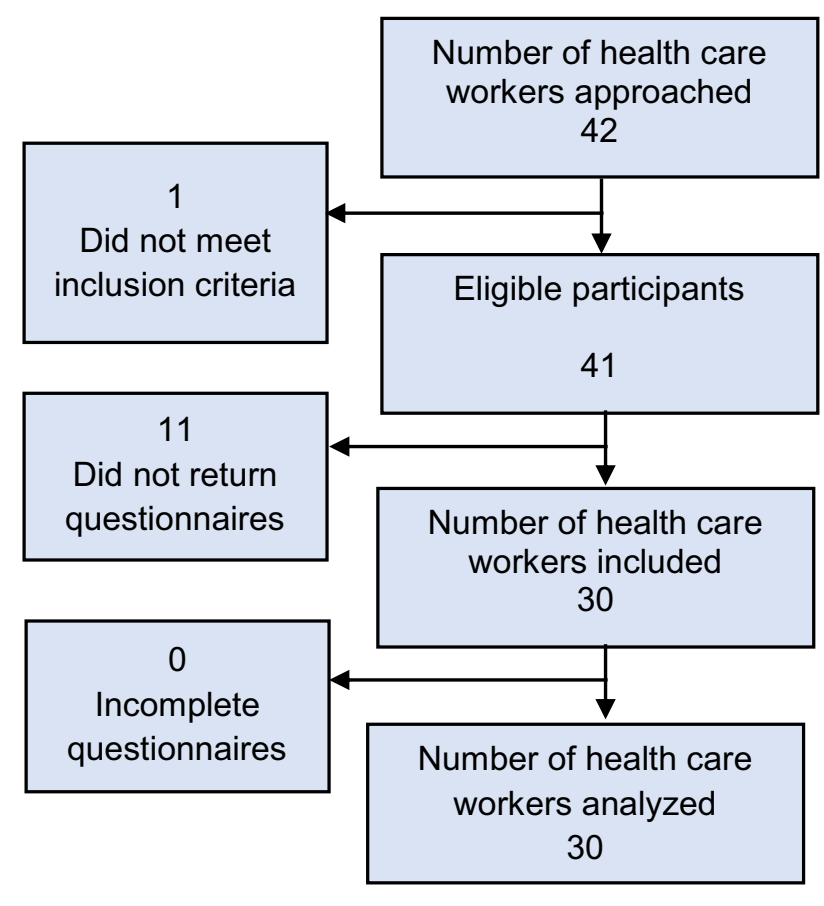

Figure I Diagram showing study recruitment.

Abbreviation: CRD, chronic respiratory disease.

\section{HCWs}

A total of $42 \mathrm{HCWs}$ were approached to participate in the survey, and 30 were recruited. Of the 12 who did not participate, one was excluded (not in regular contact with patients) and 11 did not return questionnaires (Figure 1).

\section{Participant Characteristics HCWs}

Overall, 53\% ( $\mathrm{n}=16)$ were females. The most common professional job category was hospital doctor $(n=13,43 \%)$, followed by physiotherapist $(8,27 \%)$, nurse $(4,13 \%)$, and then "other" which included pharmacists and patient counselors $(5,17 \%)$. HCW responsibilities included diagnosing (20, 66.7\%), on-going management $(18,60 \%)$, prescribing $(15,50 \%)$, outpatient clinics $(15,50 \%)$, inpatient treatment $(10,33 \%)$, non-urgent care $(8,27 \%)$, urgent assessments $(8$, $27 \%$ ), admission prevention $(7,23 \%)$, oxygen therapy $(7$, $23 \%)$, medication checks $(7,23 \%)$, primary care $(6,20 \%)$, and other (dispensing and referring patients $(5,17 \%)$ ).

\section{People with CRD}

Overall, $63 \%$ were female, the majority $(77 \%)$ were $>40$ years of age and $57 \%$ were unemployed (Table 1).

Overall, $86 \%(n=44)$ of people with CRD reported breathlessness as a major problem. A breathing problem affected $71 \%(n=36)$ of participants' ability to carry out

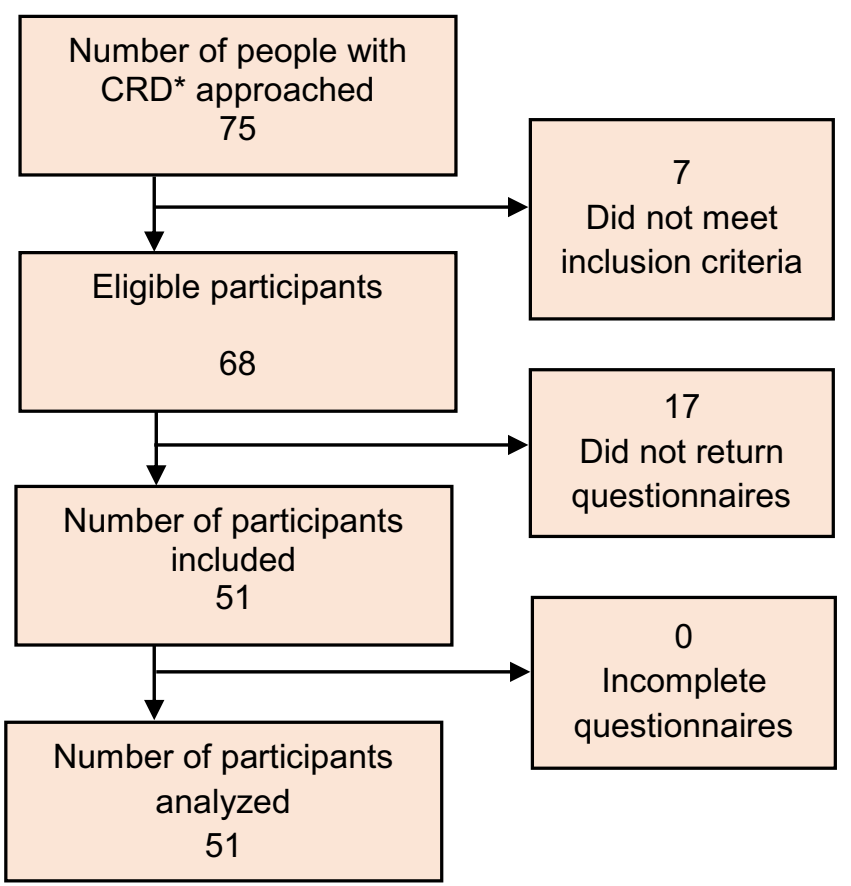

paid work and $77 \%(\mathrm{n}=39)$ of participants' ability to carry out unpaid work. A total of $69 \%(n=35)$ had missed a day of work due to breathing problems. When asked about their ability to keep up with peers when walking, $69 \%$ $(\mathrm{n}=35)$ said they were unable to and breathing problems prevented $82 \%(\mathrm{n}=42)$ from being as active as they would like. Furthermore, 63\% $(\mathrm{n}=32)$ of participants reported breathing problems prevented them from visiting friends or family as often as they would have liked.

\section{Interest in PR}

Interest in PR was also assessed and 92\% (n=47) of participants stated they would be interested in participating in a treatment that reduces breathlessness and increases activity. The preferred mode of PR was also assessed and $67 \%(\mathrm{n}=34)$ preferred a group, supervised, hospital-based PR programme that lasts between 30 minutes to 1 hour per day $(39 \%, n=20)($ Table 2$)$.

\section{HCW Survey Results}

In terms of PR being "worthwhile", 97\% $(n=29)$ answered "Yes, very much", and 3\% (n=1) answered "Yes, a little".

\section{PR Referrals by HCWs (Figure 2)}

The majority of HCWs strongly agreed that PR training at health facilities would improve PR referrals $(97 \%, n=29)$ 
Table I Characteristics of Study Participants with Chronic Respiratory Disease in Uganda. Data Presented as n (\%) Except Where Stated

\begin{tabular}{|l|l|}
\hline Participant Characteristics & Frequency \\
\hline Sex (female) & $32(63.0 \%)$ \\
\hline Age group & \\
\hline$<40$ years & $12(23.5 \%)$ \\
\hline $40-50$ years & $10(19.6 \%)$ \\
\hline $50-60$ years & $9(17.7 \%)$ \\
\hline $60-70$ years & $12(23.5 \%)$ \\
\hline $70-80$ years & $3(5.9 \%)$ \\
\hline$>80$ years & $5(9.8 \%)$ \\
\hline Age when left full-time education (median years, IQR) & $22(19-25)$ \\
\hline Employment status & \\
\hline Employed & $19(37 \%)$ \\
\hline Unemployed & $29(57 \%)$ \\
\hline Retired & $3(6 \%)$ \\
\hline
\end{tabular}

Abbreviation: IQR, Inter-Quartile Range.

Table 2 Patient Preference in Regard to the Mode of a Pulmonary Rehabilitation (PR) Programme

\begin{tabular}{|l|l|}
\hline Characteristic & $\begin{array}{l}\text { Frequency } \\
\text { (\%) }\end{array}$ \\
\hline Preferred mode of PR & \\
\hline Group, supervised, hospital-based & $34(66.7)$ \\
\hline $\begin{array}{l}\text { Group, supervised, local hospital or community } \\
\text { based }\end{array}$ & $8(15.7)$ \\
\hline Exercise and educational manual at home & $9(17.6)$ \\
\hline Web-based & $0(0)$ \\
\hline Time willing to spend in PR & $13(25.5)$ \\
\hline$<30$ minutes /day & $20(39.2)$ \\
\hline 30 minutes to I hour /day & $15(29.4)$ \\
\hline I to 2 hours /day & $3(5.9)$ \\
\hline$>2$ hours /day &
\end{tabular}

and that they were willing to work with colleagues to enable PR referrals $(90 \%, \mathrm{n}=27)$. A significant number agreed that they could easily integrate PR referral into their work $(83 \%, \mathrm{n}=25)$ and that referring patients to $\mathrm{PR}$ is worthwhile $(90 \%, \mathrm{n}=29)$.

\section{Patients Factors That Influence PR Referrals by HCWs (Figure 3 )}

The patient-based factors that strongly influence HCW to refer to PR included breathlessness affecting movement $(87 \%, n=26)$, increasing shortness of breath $(83 \%, n=25)$, low exercise tolerance $(70 \%, \mathrm{n}=21)$ and decreasing activity levels $(67 \%, \mathrm{n}=20)$.

\section{PR Referral Barriers (Figure 4)}

Regarding barriers to PR referral, the referral processbased factors that strongly influence HCWs decision not to refer included: $77 \%(\mathrm{n}=23)$ not having enough information about PR, $47 \%(n=14)$ lack time to complete the referral process, while $87 \%(\mathrm{n}=26)$ reported that being unaware of how to refer to PR had some influence in not recommending or referring patients for the service.

\section{Barriers to PR Referral}

Two categories emerged following the question "how to improve referral and uptake of PR", including the need for training and making the referral process easier. In terms of "need for training", the following responses were mentioned: continuing medical education of PR and its benefits to patients, education, having adequate information about PR, knowledge on CRD and indications for PR, and adequate knowledge about PR and the referral process. Common responses related to "making the referral process easier" included referral form or tool, guidelines and criteria to guide referral of patients for PR, streamlining the referral process for HCWs, guidance on referral and clear referral criteria.

\section{Discussion}

In this survey study, breathlessness affected people's ability to perform both paid and unpaid work, and a high number (92\%) of people with CRD expressed interest in participating in PR. In addition, a high number (77\%) of HCWs lacked enough information about PR to recommend or refer patients for this treatment; demonstrating the need for national training and education in PR to develop effective and sustainable PR referral systems in Uganda.

Breathlessness and activity limitation are common in $\mathrm{CRD}$, and are the most troublesome symptoms reported by individuals with CRD. ${ }^{22}$ This may explain why people with CRD were highly interested in attending PR in our study. In contrast, Xie et $\mathrm{al}^{23}$ found that people with CRD 
Need for training at facilities to improve PR referrals

Willing to work with colleagues to enable PR referral

Value referring patients for PR

Receive reports of PR outcomes

Can overcome PR referral difficulties

Confidence in colleagues who care for CRD patients

Can easily integrate PR referral into my work

Referring patients is part of my role

Staff agree that patient referral to PR worthwhile

Sufficient resources available to support PR referral

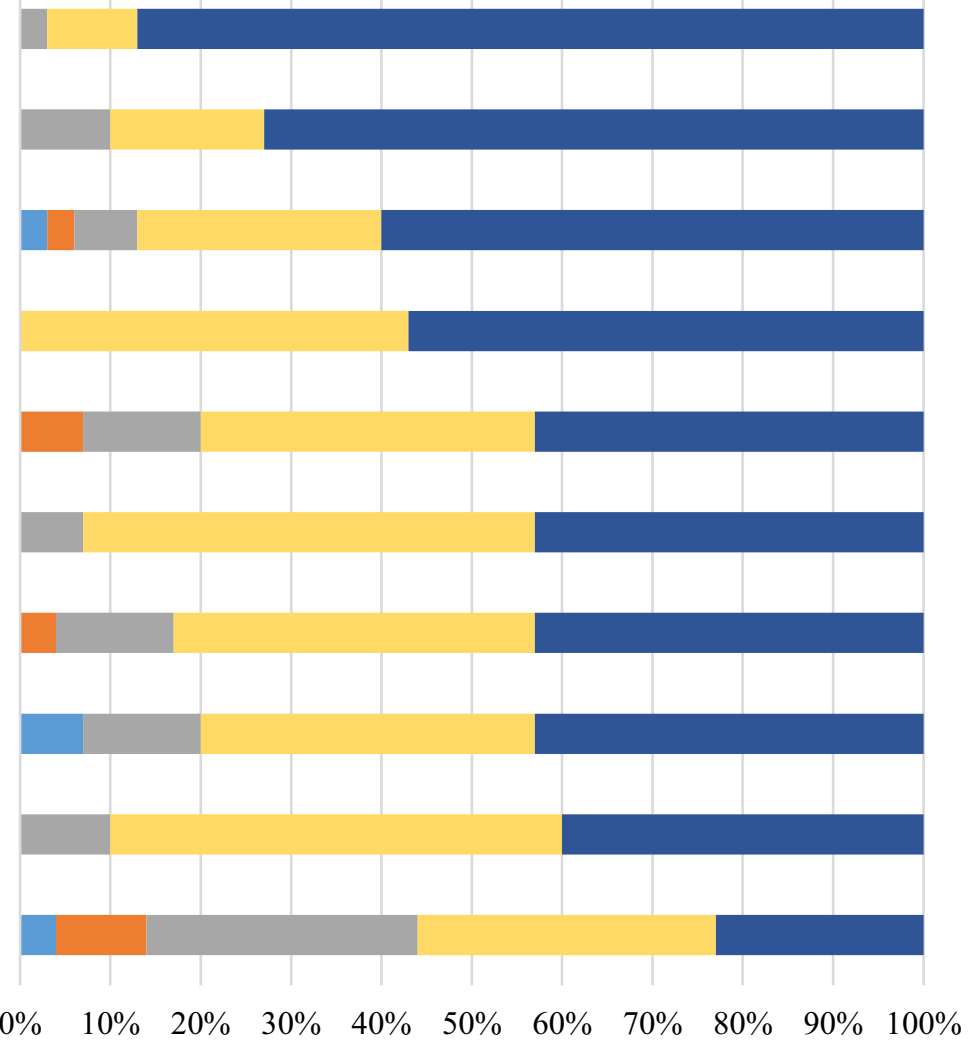

Strongly disagree $\quad$ Disagree $\quad$ Not sure $\quad$ Agree $\quad$ Strongly agree

Figure 2 Health care worker opinions on referring to Pulmonary Rehabilitation (PR). Data presented as a percentage of those that strongly disagree, disagree, not sure, agree and strongly agreed.

Abbreviation: CRD, chronic respiratory disease.

in China were less enthusiastic about participating in PR despite knowledge of benefits of PR. This was mostly attributed to patients' lack of enough information about PR. ${ }^{23}$ Although our survey did not seek to find out patients' awareness of PR, educating patients about PR and its benefits was suggested by HCWs as one of the ways of encouraging patients to take up PR in our settings. This is congruent with the ATS/ERS policy statement that recommends increasing patient knowledge and awareness of PR as key aspects of increasing implementation of PR worldwide. $^{13,15}$

This survey adds to existing knowledge of patients' opinions on PR in Uganda. In a previous qualitative study with a similar group of PTLD patients in Uganda, improvements in respiratory symptoms, functional impairment and psychological well-being were reported by patients after $\mathrm{PR} .^{24}$ This survey provides opinions of CRD patients without prior exposure to PR and shows their willingness and readiness to participate this treatment.
The gap between patients' enthusiasm for PR and the lack of awareness by HCWs of how to refer patients for this treatment in our study highlights a training need. Although the reasons for not recommending or referring people with $\mathrm{CRD}$ for PR may be multifactorial, increasing HCWs knowledge and awareness about the benefits of PR and its indications are key in setting up a successful PR programme. Clinical knowledge and skills are a critical factor in establishing and sustaining quality services, but many other factors in the work environment can directly or indirectly affect the quality of services and influence the ability of providers to apply their knowledge and skills in the services they offer. ${ }^{25}$ The lack of knowledge regarding content and benefits of PR remains a major barrier in setting up PR programs. ${ }^{26}$

Although PR in Uganda is delivered by physiotherapists, in-country formal education and training in PR is unavailable. Equipping physiotherapists across the country with PR skills is key in setting up a national PR service and potentially a training hub for the African region. Further training on how to incorporate low-cost PR within the existing physiotherapy work 


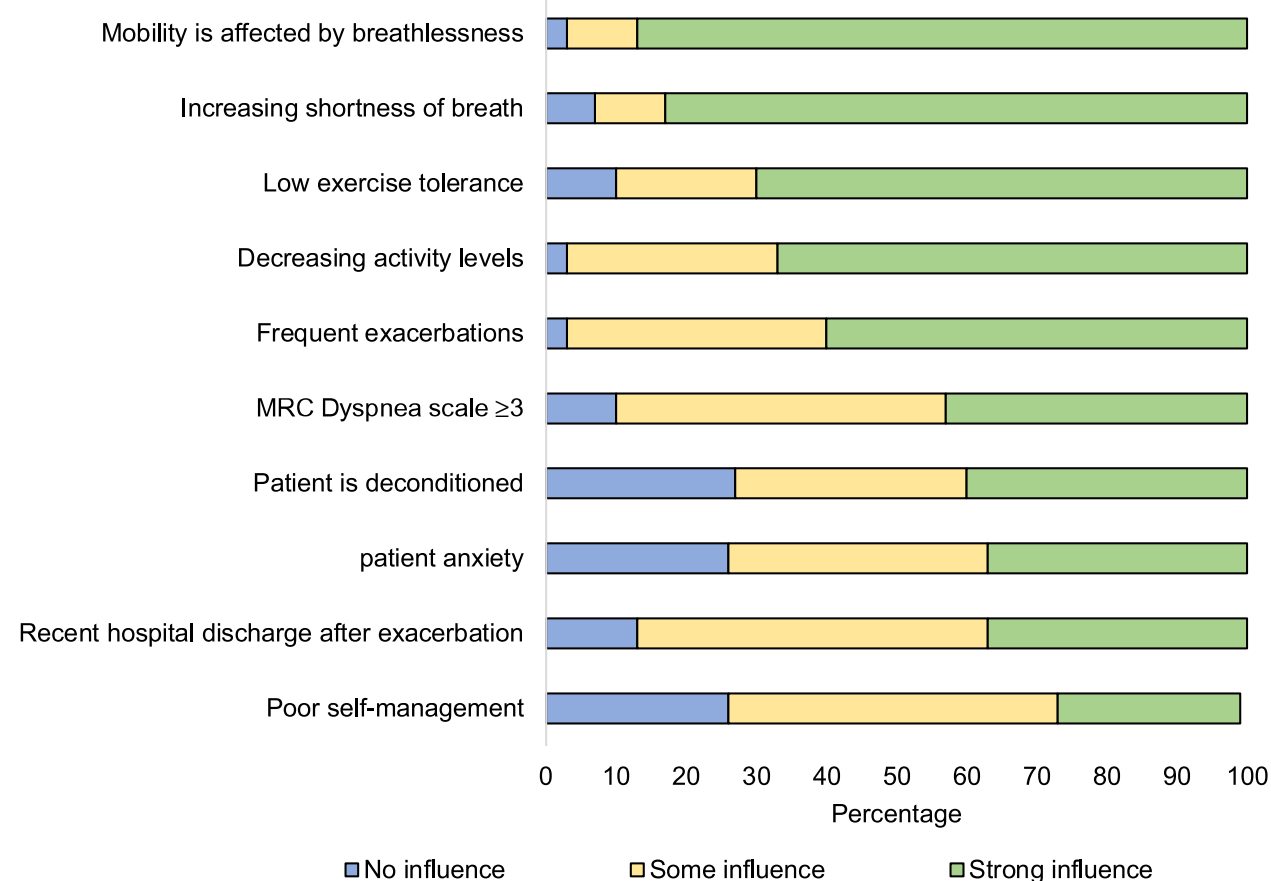

Figure 3 Patient-based factors influencing decision to refer to Pulmonary Rehabilitation, with influence graded as no, some or strong influence. Data presented as a percentage.

Abbreviation: MRC, Medical Research Council.

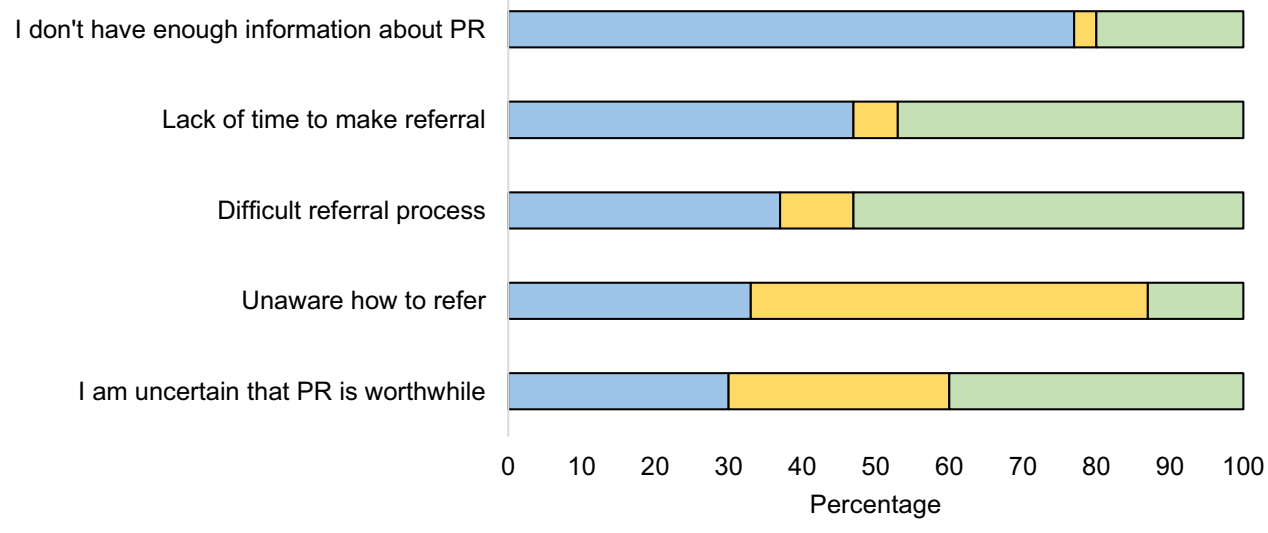

$\square$ Strong influence $\quad \square$ Some influence $\quad \square$ No influence

Figure 4 Referrer and referral process-based factors influencing decision not to refer patients to pulmonary rehabilitation (PR), with influence graded as no, some or strong influence. Data presented as a percentage.

spaces at the health facilities would have a greater impact on PR service delivery. The incorporation of PR within existing facilities, infrastructure and staffing set-up will likely reduce the cost of the start-up and sustenance of the PR service. ${ }^{27}$ This approach would potentially be cost effective in improving uptake of PR in low resource settings, where health systems are not well adapted to manage CRD. A development study of PR in Uganda showed that it was feasible to run a low-cost PR programme within existing physiotherapy facilities within the health facility set-up. ${ }^{28}$ Participants with PTLD in Uganda reported clinically important improvements in exercise capacity and quality of life. ${ }^{28}$ Countrywide and regional training on how to set up and run low-cost PR programs has the potential to improve the quality of life of people with CRD in Uganda and is crucial in achieving sustainable development goal 3 (ensuring healthy lives and promoting well-being at all ages). ${ }^{16}$ 


\section{Limitations}

This was an exploratory study with a convenience sample and a small sample size, which provides a potential selection bias. In addition, recruited participants had presented to hospital, were more likely to have good health-seeking behavior, and therefore more motivated to participate in a hospital-based PR program. Furthermore, the study would have benefited from more in-depth, qualitative exploration, but the free-text responses available highlighted important training needs and educational barriers to be addressed and further investigated. However, this survey was an exploratory study designed to obtain baseline knowledge, initial opinions and thoughts in regard to PR and therefore these limitations have little effect on the study outcomes.

\section{Generalizability}

Data were collected in a national referral hospital where adults with CRD are referred to from across Uganda. Although this provides heterogeneity of the study population, it was a convenient sample and may be subject to selection bias. Therefore, the findings may not be generalized to the target population. However, the opinions gathered during this survey provided insights into the requirements of establishing a successful PR program and referral system. A larger survey would be needed to determine if these results are generalizable to the larger population.

\section{Conclusion}

To successfully set up PR services for people with CRD in low resource settings, there is a great need for appropriately tailored training and education of prospective referrers about CRD and PR programs. This survey identified an unmet need and desire for PR among people with CRD, yet knowledge and training of health care providers is low. There is a major need for training and education of HCWs about CRD and PR. Educating patients about the benefits of PR as well as streamlining the referral process is critical in setting up a successful PR program in Uganda.

\section{Study Registration}

This study has been prospectively registered with the ISRCTN (ISRCTN44754061).

\section{Acknowledgments}

We would like to acknowledge Dr. Jonathan Fuld Addenbrookes who allowed us to modify his original survey questionnaire and who was part of the team that designed the $\mathrm{HCW}$ survey questionnaire for this study. In addition, we would like to appreciate the HCWs and the people with CRD who participated in this study.

\section{Disclosure}

The authors report no conflicts of interest in this work.

This research was funded by the National Institute for Health Research (NIHR) (17/63/20) using UK aid from the UK Government to support global health research. The views expressed in this publication are those of the authors and not necessarily those of the NIHR or the UK Department of Health and Social Care.

\section{References}

1. Labaki WW, Han MK. Chronic respiratory diseases: a global view. Lancet Respir Med. 2020;8(6):531-533. doi:10.1016/S2213-2600(20) 30157-0

2. Soriano JB, Kendrick PJ, Paulson KR, et al. Prevalence and attributable health burden of chronic respiratory diseases, 1990-2017: a systematic analysis for the Global Burden of Disease Study 2017. Lancet Respir Med. 2020;8(6):585-596. doi:10.1016/S2213-2600(20)30105-3

3. Kiadaliri AA. GBD, Global, regional, and national incidence, prevalence, and years lived with disability for 354 diseases and injuries for 195 countries and territories, 1990-2017: a systematic analysis for the Global Burden of Disease Study 2017. Lancet. 2018;392 (10159):1789-1858.

4. Kibirige D, Sanya RE, Nantanda R, et al. Availability and affordability of medicines and diagnostic tests recommended for management of asthma and chronic obstructive pulmonary disease in sub-Saharan Africa: a systematic review. Allergy Asthma Clin Immunol. 2019;15(1):14. doi:10.1186/s13223-019-0329-2

5. Lacasse Y, Wong E, Guyatt GH, et al. Meta-analysis of respiratory rehabilitation in chronic obstructive pulmonary disease. A Cochrane sys rev Eur medicophysica. 2007;43(4):475-485.

6. McCarthy B, Casey D, Devane D, et al. Pulmonary rehabilitation for chronic obstructive pulmonary disease. Cochrane Database Sys Rev. 2015;1(2):548.

7. Nici L, ZuWallack R, Wouters E, et al. On pulmonary rehabilitation and the flight of the bumblebee: the ATS/ERS Statement on Pulmonary Rehabilitation. Eur Respir J. 2006;28(3):461. doi:10.1183/09031936.06.00075106

8. Coventry PA, Hind D. Comprehensive pulmonary rehabilitation for anxiety and depression in adults with chronic obstructive pulmonary disease: systematic review and meta-analysis. J Psychosom Res. 2007;63(5):551-565. doi:10.1016/j.jpsychores.2007.08.002

9. Wouters EF, Posthuma R, Koopman M, et al. An update on pulmonary rehabilitation techniques for patients with chronic obstructive pulmonary disease. Expert Rev Respir Med. 2020;14(2):149-161. doi:10.1080/17476348.2020.1700796

10. Habib GMM, Rabinovich R, Divgi K, et al. Systematic review of clinical effectiveness, components, and delivery of pulmonary rehabilitation in low-resource settings. Npj Primary Care Respir Med. 2020;30(1):52. doi:10.1038/s41533-020-00210-y

11. Spruit MA, Singh SJ, Garvey C, et al. An official American Thoracic Society/European Respiratory Society statement: key concepts and advances in pulmonary rehabilitation. Am J Respir Crit Care Med. 2013;188(8):e13-e64. doi:10.1164/rccm.201309-1634ST 
12. Bickton FM, Fombe C, Chisati E, et al. Evidence for pulmonary rehabilitation in chronic respiratory diseases in sub-Saharan Africa: a systematic review. Int $J$ Tuberculosis Lung Dis. 2020;24 (10):991-999. doi:10.5588/ijtld.19.0526

13. Rochester CL, Vogiatzis I, Holland AE, et al. An official American Thoracic Society/European Respiratory Society Policy Statement: enhancing Implementation, Use, and Delivery of Pulmonary Rehabilitation. Am J Respir Crit Care Med. 2015;192 (11):1373-1386. doi:10.1164/rccm.201510-1966ST

14. Nici L, Donner C, Wouters E, et al. American Thoracic Society/ European Respiratory Society statement on pulmonary rehabilitation. Am J Respir Crit Care Med. 2006;173 (12):1390-1413. doi:10.1164/rccm.200508-1211ST

15. Vogiatzis I, Rochester CL, Spruit MA, et al. Increasing implementation and delivery of pulmonary rehabilitation: key messages from the new ATS/ERS policy statement. Eur Respir J. 2016;47(5):1336. doi:10.1183/13993003.02151-2015

16. Singh SJ, Halpin DMG, Salvi S, et al. Exercise and pulmonary rehabilitation for people with chronic lung disease in LMICs: challenges and opportunities. Lancet Respir Med. 2019;7(12):1002-1004. doi:10.1016/S2213-2600(19)30364-9

17. Allwood BW, Byrne A, Meghji J. et al. Post-tuberculosis lung disease: clinical review of an under-recognised global challenge. Respiration;2021. 1-13. doi:10.1159/000512531

18. Desalu OO, Onyedum CC, Adeoti AO, et al. Guideline-based COPD management in a resource-limited setting — physicians' understanding, adherence and barriers: a cross-sectional survey of internal and family medicine hospital-based physicians in Nigeria. Primary Care Respir J. 2013;22(1):79-85. doi:10.4104/pcrj.2013.00014

19. Graham BL, Steenbruggen I, Miller MR, et al. Standardization of spirometry 2019 update. An official American thoracic society and European respiratory society technical statement. Am J Respir Crit Care Med. 2019;200(8):e70-e88. doi:10.1164/rccm.201908-1590ST
20. Stenton C. The MRC breathlessness scale. Occup Med (Chic Ill). 2008;58(3):226-227. doi:10.1093/occmed/kqm162

21. Hannah DR, Lautsch BA. Counting in qualitative research: why to conduct it, when to avoid it, and when to closet it. J Management Inquiry. 2011;20(1):14-22. doi:10.1177/1056492610375988

22. O'Donnell DE. Advances in the evaluation of respiratory pathophysiology during exercise in chronic lung diseases. Front Physiol. 2017;8:82

23. Xie L, Liu Z, Hao S, et al. Assessment of knowledge, attitude, and practice towards pulmonary rehabilitation among COPD patients: a multicenter and cross-sectional survey in China. Respir Med. 2020;174:106198. doi:10.1016/j.rmed.2020.106198

24. Jones R, Muyinda H, Nyakoojo G, et al. Does pulmonary rehabilitation alter patients' experiences of living with chronic respiratory disease? A qualitative study. Int J Chron Obstruct Pulmon Dis. 2018;13:2375-2385. doi:10.2147/COPD.S165623

25. Nelson D, Dufour W. Transfer of learning: A guide for strengthening the performance of health care workers. Education Resources Information Center; 2002.

26. Alsubaiei ME, Cafarella P, Frith $P$, et al. Barriers for setting up a pulmonary rehabilitation program in the Eastern Province of Saudi Arabia. Ann Thorac Med. 2016;11(2):121. doi:10.4103/18171737.180028

27. Cox NS, Oliveira CC, Lahham A, et al. Pulmonary rehabilitation referral and participation are commonly influenced by environment, knowledge, and beliefs about consequences: a systematic review using the theoretical domains framework. J Physiother. 2017;63 (2):84-93. doi:10.1016/j.jphys.2017.02.002

28. Jones R, Kirenga BJ, Katagira W, et al. A pre-post intervention study of pulmonary rehabilitation for adults with post-tuberculosis lung disease in Uganda. Int $J$ Chron Obstruct Pulmon Dis. 2017;12:3533. doi:10.2147/COPD.S146659

\section{Publish your work in this journal}

The International Journal of COPD is an international, peer-reviewed journal of therapeutics and pharmacology focusing on concise rapid reporting of clinical studies and reviews in COPD. Special focus is given to the pathophysiological processes underlying the disease, intervention programs, patient focused education, and self management protocols. This journal is indexed on PubMed Central, MedLine and CAS. The manuscript management system is completely online and includes a very quick and fair peer-review system, which is all easy to use. Visit http://www.dovepress.com/testimonials.php to read real quotes from published authors. 